\title{
Transanal single-port low anterior resection in a cadaver model
}

\author{
Alyssa D. Fajardo $\cdot$ Steven R. Hunt • \\ James W. Fleshman • Matthew G. Mutch
}

Received: 29 September 2009/Accepted: 27 November 2009/Published online: 7 January 2010

(C) Springer Science+Business Media, LLC 2010

\begin{abstract}
Background Natural orifice transluminal endoscopic surgery (NOTES), a recent development in the field of minimally invasive surgery, provides theoretical advantages over open and laparoscopic surgery. Challenges with the use of NOTES for colon resections include the need to extract a large specimen and perform an intestinal anastomosis. A transanal single-port laparoscopic proctectomy uses the potential advantages of NOTES yet provides easy specimen extraction and anastomosis.

Methods Fresh frozen then thawed human cadavers were used. Access was obtained via transanal mucosectomy, and a transanal endoscopic microsurgery (TEM) system was used to perform the proctectomy once the procedure was above the pelvic floor. After the peritoneal cavity was entered, a hand port was placed through the sphincter and above the pelvic floor. The left colon was mobilized using two 5-mm working ports and a 10-mm camera port for the flexible-tipped laparoscope that were placed through the hand port. The specimen was removed transanally.

Results The critical steps for an oncologic resection of the rectum were achieved using a combination of TEM and laparoscopic instrumentation transanally. The rectum and
\end{abstract}

Electronic supplementary material The online version of this article (doi:10.1007/s00464-009-0838-z) contains supplementary material, which is available to authorized users.

A. D. Fajardo · S. R. Hunt · J. W. Fleshman · M. G. Mutch ( ()

Department of Surgery, Section of Colon and Rectal Surgery,

Washington University in St. Louis School of Medicine,

660 S. Euclid Avenue, Campus Box 8109, Saint Louis,

MO 63110, USA

e-mail: mutchm@wustl.edu

A. D. Fajardo

e-mail: fajardoaly@wudosis.wustl.edu mesorectum were mobilized. The left ureter was identified and preserved, the inferior mesenteric artery (IMA) ligated at its origin, the inferior mesenteric vein ligated near the ligament of Treitz, the proximal colon mobilized for transanal extraction, the specimen resected proximal to the ligated IMA, and a hand-sewn coloanal anastomosis performed. Postresection examination showed preservation of the hypogastric nerves and an intact mesorectum. Challenges included maneuverability around the sacral promontory and length of the instruments. However, this was easily overcome by the use of long reticulating instruments and a flexible-tipped laparoscope.

Conclusions Transanal single-port low anterior resection using TEM and laparoscopic techniques is feasible and can maintain the principles of an oncologic resection.

Keywords Laparoscopy - Natural orifice surgery · Proctectomy $\cdot$ Single port

Disclosures Steven R. Hunt receives an honorarium as a course instructor for Storz, Applied Medical, and Ethicon. James W. Fleshman receives research support/grants from Ethicon, NITI, LifeCell, Applied Medical, Helsinn-(Sapphire), Innocoll, and Novadaq Technologies, as well as an honorarium from Helsinn-(Sapphire), Innocoll, and Genzyme for consulting. Matthew Mutch receives an honorarium from Applied Medical, Ethicon, and Covidien. Alyssa D. Fajardo has no conflicts of interest or financial ties to disclose. 